\title{
Non obesity, autoimmunity and the missing pieces of diabetes in the adult
}

\author{
Krishna G. Seshadri
}

Received: 13 April 2013 /Accepted: 15 April 2013 /Published online: 21 June 2013

(C) Research Society for Study of Diabetes in India 2013

The unholy alliance between diabetes and obesity has dominated the metabolic literature space. Even a cursory search of indexed articles with the tag obesity and diabetes in the title or abstract for the period January 2012 through January 2013 yielded 4181 articles. New data on non obese diabetes is indeed therefore welcome. In this small study, Chandni et al. [1] report the spectrum of diabetes in 31 patients with an average BMI of $18.7 \mathrm{~kg} / \mathrm{m}^{2}$ and waist circumference of $74 \mathrm{~cm}$. Of these $45.1 \%$ had subnormal C peptide and approximately half had either positive GAD $(25.8 \%)$, islet cell antibody (22.5\%) or both (9.7\%). [1] The authors mention in passing that $\mathrm{C}$ peptide levels were lower in these patients.

Autoimmune diabetes is characterized by specific autoantibodies such as GAD autoantibodies (GADA), insulinomaassociated antigen-2 autoantibodies (IA-2A) or zinc-transporter 8 autoantibodies (ZnT8A). Type 1 diabetes is the most prevalent form of diabetes in children but also occurs in adults. The majority of patients with type 1 diabetes have autoantibodies in their peripheral blood, which can predict the future onset of the disease [2].

Latent Autoimmune Diabetes in Adults (LADA) sits between type 1 diabetes and type 2 diabetes, in a manner of saying. Depending on the geographic area, up to $20 \%$ of adult diabetics may have LADA [3]. Similar studies from India peg the incidence to be around $5 \%$ [4] The typical features include younger age of onset, non obese, lack of family history, early requirement of insulin, low C-peptide levels and positive antibodies particularly GAD [3]. There are significant variations to this decade old norm. For instance family history and familial clustering have since been reported [5]. Female preponderance, higher HDL and lower

\footnotetext{
K. G. Seshadri $(\bowtie)$

Endocrinology Diabetes and Metabolism,

Sri Ramachandra University, Chennai, India

e-mail: krishnagseshadri@gmail.com
}

triglycerides have been reported [6]. The percentage and distribution of antibodies reported vary in differing geographic corners of the world and possibly even in different parts of the country [7-9]. The risk and consequences of metabolic syndrome may also be lower. GAD positivity appears to strongly predict diabetes of this kind. In a large population study from Finland, the incidence of diabetes was highest in subjects with the highest quartile of GAD antibodies. The younger the age of GAD positivity, the shorter is the progression.

In the clinic - LADA is still a clinical diagnosis; Clinicians suspect the condition in patients hitherto diagnosed as type 2 but requiring early transfer to insulin. Antibodies are seldom measured due to many factors but are the way to diagnosis. It is possible that this "common sense diagnosis" is fallacious as time to initiation of insulin can vary significantly [10]. It also appears that there is a gradation of clinical and biochemical features in patients, in which GAD antibody titer is a continuous variable, ranging from high through low titer to GAD negativity, without any clear distinction apart from the presence or absence of GAD antibody [6]. The phenotype of LADA that we rely on in clinical practice appears to represent, only a spectral end of a continua of autoimmune diabetes and must be thus viewed.

Decades after the discovery of this subtype it is unclear if it has the same course of the type 1 or type 2 diabetes; does it chart an independent course in-terms of outcomes and complications? Even more important: is there a clinical, biochemical or demographic signal that predicts this outcome?

The study of Chandni et al. [1]. is important despite the usual methodologic limitations of a single center cross sectional study. Indeed, this study allows for no conclusions or implications other than highlighting the need to fish out other kinds of diabetes when wading in a sea of type 2 diabetes. In patients with relative (or absolute) insulin deficiency what were the other antibodies present? Did the other half who were $\mathrm{C}$ peptide sufficient have insulin resistance? 
As laudable as the effort of the authors in attempting the study is, more questions than answers emerge. What the study spotlights therefore is the unmet need for a collaborative well funded, multi-centric study that examines the clinical profiles, family clustering antibody positivity and heterogeneity, antibody phenotype correlation and response to therapy. And we hope some prospective researchers find this editorial nudging them to do just that.

\section{References}

1. Chandni R, Binoy JP, Udayabhaskaran V, Ramamoorthy KP. A study of non-obese diabetes mellitus in adults in a tertiary care hospital in Kerala, India. Int J Diabetes Dev Ctries. 2013. doi:10.1007/s13410-013-0113-7

2. Bluestone JA, Herold K, Eisenbarth G. Genetics, pathogenesis and clinical interventions in type 1 diabetes. Nature. 2010;464:1293-300.

3. Landin-Olsson M. Latent autoimmune diabetes in adults. Ann N Y Acad Sci. 2002;958:112-6.
4. Brahmkshatriya PP, Mehta AA, Saboo BD, Goyal RK. "Characteristics and Prevalence of Latent Autoimmune Diabetes in Adults (LADA).” ISRN Pharmacology. 2012; 2012, Article ID 580202, 8 pages. doi:10.5402/2012/580202.

5. Castleden HA, Shields B, Bingley PJ, et al. GAD antibodies in probands and their relatives in a cohort clinically selected for type 2 diabetes. Diabet Med. 2006;23:834-8.

6. Hawa MI, Kolb H, Schloot N, Beyan H, Paschou SA, Buzzetti R, et al. Adult-onset autoimmune diabetes in Europe is prevalent with a broad clinical phenotype: action LADA 7. Diabetes Care. 2013;36:908-13.

7. Kanungo A, Sanjeevi CB. IA-2 autoantibodies are predominant in latent autoimmune diabetes in adults patients from Eastern India. Ann N Y Acad Sci. 2003;1005:390-4.

8. Gupta M, Tandon N, Shtauvere-Brameus A, Sanjeevi CB. ICA12 autoantibodies are associated with non-DR3/non-DR4 in patients with latent autoimmune diabetes in adults from Northern India. Ann N Y Acad Sci. 2002;958:329-32.

9. Sanjeevi CB, Balaji M, Balaji V, Seshiah V. Autoantibodies to GAD65 and IA-2 antibodies are increased, but not tissue transglutaminase (TTG-Ab) in type 2 diabetes mellitus (T2DM) patients from South India. Ann N Y Acad Sci. 2003;1005:387-9.

10. Brophy S, Yderstræde K, Mauricio D, Hunter S, Hawa M, Pozzilli $\mathrm{P}$, et al. Time to insulin initiation cannot be used in defining latent autoimmune diabetes in adults. Diabetes Care. 2008;31:439-41. 